\title{
Lyapunov-type inequalities for nonlinear
} fractional differential equations and systems involving Caputo-type fractional derivatives

\author{
Mohamed Jleli ${ }^{1}$, Bessem Samet ${ }^{1 *}$ (ID and Yong Zhou ${ }^{2,3}$
}

"Correspondence:

bsamet@ksu.edu.sa

'Department of Mathematics,

College of Science, King Saud

University, Riyadh, Saudi Arabia

Full list of author information is

available at the end of the article

\begin{abstract}
A Lyapunov-type inequality is derived for a nonlinear fractional boundary value problem involving Caputo-type fractional derivative. The obtained inequality provides a necessary condition for the existence of nontrivial solutions to the considered problem. Next, we extend our study to the case of systems.
\end{abstract}

MSC: 26D10; 34A08; 26A33

Keywords: Lyapunov-type inequalities; Caputo-type fractional derivative; Systems

\section{Introduction}

In this paper, we are concerned with the nonlinear fractional boundary value problem

$$
\left\{\begin{array}{l}
\left({ }_{*}^{\rho} D_{a^{+}}^{\alpha} u\right)(t)+\varphi(t, u)=0, \quad a<t<b, \\
u(a)=u(b)=0,
\end{array}\right.
$$

where $a, b \in \mathbb{R}, 0<a<b, \rho>0,1<\alpha<2,{ }_{*}^{\rho} D_{a^{+}}^{\alpha}$ is the (left-sided) Caputo-type fractional derivative of order $\alpha$ and $\varphi:[a, b] \times C([a, b]) \rightarrow \mathbb{R}$ is a given function. We establish a Lyapunov-type inequality for the considered problem. Such inequality provides a necessary condition for the existence of nontrivial solutions to (1.1). Next, we study the system

$$
\left\{\begin{array}{l}
\left({ }_{*}^{\rho} D_{a^{+}}^{\alpha} u\right)(t)+\varphi(t, u, v)=0, \quad a<t<b, \\
\left({ }_{*}^{\rho} D_{a^{+}}^{\alpha} v\right)(t)+\psi(t, u, v)=0, \quad a<t<b, \\
u(a)=u(b)=v(a)=v(b)=0,
\end{array}\right.
$$

where $a, b \in \mathbb{R}, 0<a<b, \rho>0,1<\alpha<2$ and $\varphi, \psi:[a, b] \times C([a, b]) \times C([a, b]) \rightarrow \mathbb{R}$ are given functions. Let us mention some motivations for studying problems as in (1.1) and (1.2).

The classical Lyapunov inequality [23] is related to the second order linear differential equation

$$
u^{\prime \prime}(t)+q(t) u(t)=0, \quad a<t<b,
$$

(c) The Author(s) 2019. This article is distributed under the terms of the Creative Commons Attribution 4.0 International License (http://creativecommons.org/licenses/by/4.0/), which permits unrestricted use, distribution, and reproduction in any medium, provided you give appropriate credit to the original author(s) and the source, provide a link to the Creative Commons license, and indicate if changes were made. 
under the boundary conditions

$$
u(a)=u(b)=0,
$$

where $a, b \in \mathbb{R}, a<b$ and $q \in C([a, b])$. It shows that if (1.3)-(1.4) admits a nontrivial solution $u \in C^{2}([a, b])$, then

$$
\int_{a}^{b}|q(t)| d t>\frac{4}{b-a}
$$

Inequality (1.5) has found many practical applications in the theory of differential equations (see, for example, $[2,5,22,32,34]$ and the references therein). For more improvements and generalizations of (1.5), we refer to $[1,4,6,10,13,24,28-30,33]$ and the references therein. On the other hand, due to the great attention which has been given in these last years to fractional calculus, several results related to the study of Lyapunovtype inequalities for fractional differential equations were obtained. The first work in this direction is due to Ferreira [11], where the standard derivative $u^{\prime \prime}$ in (1.3) is replaced by $D_{a^{+}}^{\alpha} u$, the Riemann-Liouville fractional derivative of order $1<\alpha<2$ of $u$. Next, in [12], the same author studied the fractional boundary value problem

$$
\left\{\begin{array}{l}
\left({ }^{C} D_{a^{+}}^{\alpha} u\right)(t)+q(t) u(t)=0, \quad a<t<b, \\
u(a)=u(b)=0,
\end{array}\right.
$$

where $1<\alpha<2,{ }^{C} D_{a^{+}}^{\alpha}$ is the Caputo fractional derivative of order $\alpha$ and $q \in C([a, b])$. The main result in [12] is the following: Let $u \in C^{2}([a, b])$ be a nontrivial solution to (1.6), then

$$
\int_{a}^{b}|q(t)| d t>\frac{\alpha^{\alpha} \Gamma(\alpha)}{[(\alpha-1)(b-a)]^{\alpha-1}} .
$$

Note that in the limit case $\alpha \rightarrow 2^{-}$, (1.6) reduces to (1.3). Moreover, observe that in the limit case $\alpha \rightarrow 2^{-}$, (1.7) reduces to (1.5). For other contributions related to Lyapunovtype inequalities for fractional differential equations, we refer to $[3,7,8,14-18,26,27]$ and the references therein. Motivated by the above cited works, a study of Lyapunov-type inequalities for problems (1.1) and (1.2) is performed in this paper.

The paper is organized as follows. In Sect. 2, we provide some preliminary results related to fractional calculus and operator theory. In Sect. 3, a Lyapunov-type inequality is established for problem (1.1) and some particular cases are discussed. In Sect. 4, we derive a Lyapunov-type inequality for system (1.2) and discuss some special cases.

\section{Preliminaries}

Let $a, b \in \mathbb{R}$ be such that $0<a<b$. We refer the reader to Samko et al. [31] for the following concepts.

Definition 2.1 Let $\theta>0$. The (left-sided) Riemann-Liouville fractional integral of order $\theta$ of a function $f \in C([a, b])$ is given by

$$
\left(I_{a^{+}}^{\theta} f\right)(t)=\frac{1}{\Gamma(\theta)} \int_{a}^{t}(t-s)^{\theta-1} f(s) d s, \quad a \leq t \leq b,
$$

where $\Gamma$ is the Gamma function. 
Definition 2.2 Let $n-1<\alpha<n$, where $n \geq 1$ is a natural number. The (left-sided) Caputo fractional derivative of order $\alpha$ of a function $f \in C^{n}([a, b])$ is given by

$$
\left({ }^{C} D_{a^{+}}^{\alpha} f\right)(t)=\left(I_{a^{+}}^{n-\alpha} f^{(n)}\right)(t), \quad a \leq t \leq b,
$$

i.e.,

$$
\left({ }^{C} D_{a^{+}}^{\alpha} f\right)(t)=\frac{1}{\Gamma(n-\alpha)} \int_{a}^{t}(t-s)^{n-\alpha-1} f^{(n)}(s) d s, \quad a \leq t \leq b .
$$

In [20] (see also [21]), Katugampola introduced the following fractional integral operator, which depends on a certain parameter $\rho>0$.

Definition 2.3 Let $\rho>0$ and $\theta>0$. The (left-sided) Katugampola fractional integral of order $\theta$ of a function $f \in C([a, b])$ is given by

$$
\left({ }^{\rho} I_{a^{+}}^{\theta} f\right)(t)=\frac{\rho^{1-\theta}}{\Gamma(\theta)} \int_{a}^{t} s^{\rho-1}\left(t^{\rho}-s^{\rho}\right)^{\theta-1} f(s) d s, \quad a \leq t \leq b .
$$

Using the above definition, D.S. Oliveira and E.C. de Oliveira [25] introduced a Caputotype fractional derivative operator as follows.

Definition 2.4 Let $\rho>0$ and $n-1<\alpha<n$, where $n \geq 1$ is a natural number. The (leftsided) Caputo-type fractional derivative of order $\alpha$ of a function $f \in C^{n}([a, b])$ is given by

$$
\left({ }_{*}^{\rho} D_{a^{+}}^{\alpha} f\right)(t)=\left({ }^{\rho} I_{a^{+}}^{n-\alpha}\left(t^{1-\rho} \frac{d}{d t}\right)^{n} f\right)(t), \quad a \leq t \leq b,
$$

i.e.,

$$
\left({ }_{*}^{\rho} D_{a^{+}}^{\alpha} f\right)(t)=\frac{\rho^{1-n+\alpha}}{\Gamma(n-\alpha)} \int_{a}^{t} s^{\rho-1}\left(t^{\rho}-s^{\rho}\right)^{n-\alpha-1}\left(s^{1-\rho} \frac{d}{d s}\right)^{n} f(s) d s, \quad a \leq t \leq b .
$$

Observe that for $\rho=1$ we have

$$
{ }_{*}^{\rho} D_{a^{+}}^{\alpha} f={ }^{C} D_{a^{+}}^{\alpha} f \text {. }
$$

Moreover, we have (see [25])

$$
\lim _{\rho \rightarrow 0^{+}}\left({ }_{*}^{\rho} D_{a^{+}}^{\alpha} f\right)(t)=\left({ }^{\mathrm{CH}} D_{a^{+}}^{\alpha} f\right)(t), \quad a \leq t \leq b,
$$

where ${ }^{\mathrm{CH}} D_{a^{+}}^{\alpha}$ is the Caputo-Hadamard fractional derivative of order $\alpha$ given by

$$
\left({ }^{\mathrm{CH}} D_{a^{+}}^{\alpha} f\right)(t)=\frac{1}{\Gamma(n-\alpha)} \int_{a}^{t}\left(\ln \frac{t}{s}\right)^{n-\alpha-1}\left(s \frac{d}{d s}\right)^{n} f(s) d s, \quad a \leq t \leq b .
$$

Further, let us fix $\rho>0$. We introduce the mapping

$$
T: C([a, b]) \rightarrow C\left(\left[a^{\rho}, b^{\rho}\right]\right)
$$


defined by

$$
(T f)(z)=f\left(z^{\frac{1}{\rho}}\right), \quad a^{\rho} \leq z \leq b^{\rho},
$$

for all $f \in C([a, b])$. Observe that the mapping $T$ is invertible and its inverse is the mapping

$$
T^{-1}: C\left(\left[a^{\rho}, b^{\rho}\right]\right) \rightarrow C([a, b])
$$

defined by

$$
\left(T^{-1} g\right)(t)=g\left(t^{\rho}\right), \quad a \leq t \leq b,
$$

for all $g \in C\left(\left[a^{\rho}, b^{\rho}\right]\right)$.

The following lemma will play an essential role in the proofs of our main results.

Lemma 2.1 Let $n-1<\alpha<n$, where $n \geq 1$ is a natural number. For any function $f \in$ $C^{n}([a, b])$, we have

$$
\left({ }_{*}^{\rho} D_{a^{+}}^{\alpha} f\right)(t)=\rho^{\alpha}\left({ }^{C} D_{a^{\rho+}}^{\alpha} T f\right)\left(t^{\rho}\right), \quad a \leq t \leq b .
$$

Proof For $a \leq s \leq b$, let us consider the change of variable

$$
\widetilde{s}=s^{\rho} .
$$

Using the chain rule, we get

$$
\frac{d}{d s}=\frac{d}{d \widetilde{s}} \frac{d \widetilde{s}}{d s}=\rho s^{\rho-1} \frac{d}{d \widetilde{s}} .
$$

Hence, for $a \leq t \leq b$, we have

$$
\begin{aligned}
\left({ }_{*}^{\rho} D_{a^{+}}^{\alpha} f\right)(t) & =\frac{\rho^{1-n+\alpha}}{\Gamma(n-\alpha)} \int_{a}^{t} s^{\rho-1}\left(t^{\rho}-s^{\rho}\right)^{n-\alpha-1}\left(s^{1-\rho} \frac{d}{d s}\right)^{n} f(s) d s \\
& =\frac{\rho^{\alpha}}{\Gamma(n-\alpha)} \int_{a^{\rho}}^{t^{\rho}}\left(t^{\rho}-\widetilde{s}\right)^{n-\alpha-1}\left(\frac{d}{d \widetilde{s}}\right)^{n} f\left(\widetilde{s}^{\frac{1}{\rho}}\right) d \widetilde{s} \\
& =\frac{\rho^{\alpha}}{\Gamma(n-\alpha)} \int_{a^{\rho}}^{t^{\rho}}\left(t^{\rho}-\widetilde{s}\right)^{n-\alpha-1}\left(\frac{d}{d \widetilde{s}}\right)^{n}(T f)(\widetilde{s}) d \widetilde{s} \\
& =\rho^{\alpha}\left({ }^{C} D_{a^{\rho}}^{\alpha} T f\right)\left(t^{\rho}\right) .
\end{aligned}
$$

The proof of the following lemma can be found in [12].

Lemma 2.2 Let $h \in C([A, B])$, where $A, B \in \mathbb{R}, A<B$. Let $v \in C^{2}([A, B])$ be a solution to the fractional boundary value problem

$$
\left\{\begin{array}{l}
\left({ }^{C} D_{A^{+}}^{\alpha} v\right)(t)+h(t)=0, \quad a<t<b, \\
v(A)=v(B)=0
\end{array}\right.
$$


where $1<\alpha<2$. Then

$$
v(t)=\int_{A}^{B} G(t, s) h(s) d s, \quad A \leq t \leq b,
$$

where

$$
G(t, s)=\frac{1}{\Gamma(\alpha)} \begin{cases}\frac{(t-A)(B-s)^{\alpha-1}}{B-A}-(t-s)^{\alpha-1}, & A \leq s \leq t \leq B, \\ \frac{(t-A)(B-s)^{\alpha-1}}{B-A}, & A \leq t \leq s \leq B .\end{cases}
$$

Moreover, we have

$$
|G(t, s)| \leq \frac{[(\alpha-1)(B-A)]^{\alpha-1}}{\alpha^{\alpha} \Gamma(\alpha)}, \quad(t, s) \in[A, B] \times[A, B] .
$$

Further, let us recall some notions of operator theory that will be used later (see, for example, $[9,19])$.

Let $N \geq 1$ be a given natural number. We introduce in $\mathbb{R}^{N}$ the partial order $\preceq_{N}$ defined by

$$
\vec{x}=\left(\begin{array}{c}
x_{1} \\
x_{2} \\
\vdots \\
x_{N}
\end{array}\right) \preceq_{N} \vec{y}=\left(\begin{array}{c}
y_{1} \\
y_{2} \\
\vdots \\
y_{N}
\end{array}\right) \Longleftrightarrow x_{i} \leq y_{i}, \quad i=1,2, \ldots, N .
$$

The zero vector of $\mathbb{R}^{N}$ is denoted by $0_{\mathbb{R}^{N}}$. We denote by $\|\cdot\|_{N}$ the Euclidean norm in $\mathbb{R}^{N}$, i.e.,

$$
\|\vec{x}\|_{N}=\left(x_{1}^{2}+x_{2}^{2}+\cdots+x_{N}^{2}\right)^{\frac{1}{2}}, \quad \vec{x}=\left(\begin{array}{c}
x_{1} \\
x_{2} \\
\vdots \\
x_{N}
\end{array}\right) \in \mathbb{R}^{N} .
$$

Lemma 2.3 Let

$$
\vec{x}=\left(\begin{array}{c}
x_{1} \\
x_{2} \\
\vdots \\
x_{N}
\end{array}\right), \quad \vec{y}=\left(\begin{array}{c}
y_{1} \\
y_{2} \\
\vdots \\
y_{N}
\end{array}\right) \in \mathbb{R}^{N}
$$

be such that

$$
0_{\mathbb{R}^{N}} \preceq_{N} \vec{x} \preceq_{N} \vec{y} .
$$

Then

$\|\vec{x}\|_{N} \leq\|\vec{y}\|_{N}$ 
Let $\mathcal{M}_{N}(\mathbb{R})$ be the set of square matrices of size $N$ with entries in $\mathbb{R}$. We denote by $\mathcal{M}_{N}\left(\mathbb{R}_{+}\right)$the subset of $\mathcal{M}_{N}(\mathbb{R})$ with positive entries. We endow $\mathcal{M}_{N}(\mathbb{R})$ with the subordinate matrix norm

$$
\|A\|_{\mathcal{M}_{N}}=\sup _{\vec{x} \in \mathbb{R}^{N}, \vec{x} \neq 0_{\mathbb{R}^{N}}} \frac{\|A \vec{x}\|_{N}}{\|\vec{x}\|_{N}}, \quad A \in \mathcal{M}_{N}(\mathbb{R}) .
$$

Given $A \in \mathcal{M}_{N}(\mathbb{R})$, we denote by $r(A)$ its spectral radius, i.e.,

$$
r(A)=\max \left\{\left|\lambda_{i}(A)\right|: i=1,2, \ldots, N\right\}
$$

where $\lambda_{i}(A), i=1,2, \ldots, N$, are the (real or complex) eigenvalues of matrix $A$.

Lemma 2.4 Let $A \in \mathcal{M}_{N}(\mathbb{R})$. Then

$$
r(A)<1 \Longleftrightarrow \lim _{n \rightarrow \infty}\left\|A^{n}\right\|_{\mathcal{M}_{N}}=0
$$

Further, we shall prove the following property, which will be used later.

Lemma 2.5 Let $A \in \mathcal{M}_{N}\left(\mathbb{R}_{+}\right)$and $\vec{x} \in \mathbb{R}^{N}, \vec{x} \neq 0_{\mathbb{R}^{N}}$. If

$$
0_{\mathbb{R}^{N}} \preceq_{N} \vec{x} \preceq_{N} A \vec{x},
$$

then

$$
r(A) \geq 1
$$

Proof Using (2.6) and the fact that $A \in \mathcal{M}_{N}\left(\mathbb{R}_{+}\right)$, for all natural numbers $n \geq 1$, we obtain

$$
0_{\mathbb{R}^{N}} \preceq_{N} \vec{x} \preceq_{N} A^{n} \vec{x}
$$

Therefore, by Lemma 2.3, we have

$$
\|\vec{x}\|_{N} \leq\left\|A^{n} \vec{x}\right\|_{N} \leq\left\|A^{n}\right\|_{\mathcal{M}_{N}}\|\vec{x}\|_{N}, \quad n \geq 1
$$

Since $\vec{x} \neq 0_{\mathbb{R}^{N}}$, dividing by $\|\vec{x}\|_{N}$, we get

$$
\left\|A^{n}\right\|_{\mathcal{M}_{N}} \geq 1, \quad n \geq 1
$$

Finally, using Lemma 2.4, (2.7) follows.

In the sequel, the functional space $C([a, b])$ is equipped with the norm

$$
\|u\|_{\infty}=\max _{a \leq t \leq b}|u(t)|, \quad u \in C([a, b]) .
$$




\section{A Lyapunov-type inequality for problem (1.1)}

Problem (1.1) is investigated under the following assumption: The function

$$
\varphi:[a, b] \times C([a, b]) \rightarrow \mathbb{R}
$$

is continuous and satisfies

$$
|\varphi(t, h)| \leq w(t)\|h\|_{\infty}, \quad(t, h) \in[a, b] \times C([a, b]),
$$

where $w \in C([a, b])$.

Observe that by (3.1), the zero function is a solution to (1.1).

Our main result in this section is the following.

Theorem 3.1 Let $u \in C^{2}([a, b])$ be a nontrivial solution to (1.1). Then

$$
\int_{a}^{b} w(t) t^{\rho-1} d t \geq \frac{\rho^{\alpha-1} \alpha^{\alpha} \Gamma(\alpha)}{\left[(\alpha-1)\left(b^{\rho}-a^{\rho}\right)\right]^{\alpha-1}}
$$

Proof Let $u \in C^{2}([a, b])$ be a nontrivial solution to (1.1). Let us introduce the function $v \in C^{2}\left(\left[a^{\rho}, b^{\rho}\right]\right)$ given by

$$
v=T u
$$

where $T$ is the mapping defined by (2.3). Using Lemma 2.1, we deduce that $v$ is a solution to

$$
\left\{\begin{array}{l}
\rho^{\alpha}\left({ }^{C} D_{a^{\rho+}}^{\alpha} v\right)\left(t^{\rho}\right)+\varphi\left(t, T^{-1} v\right)=0, \quad a<t<b, \\
v\left(a^{\rho}\right)=v\left(b^{\rho}\right)=0
\end{array}\right.
$$

where $T^{-1}$ is given by (2.4). Using the change of variable $z=t^{\rho}, a \leq t \leq b$, we deduce that $v$ is a solution to

$$
\left\{\begin{array}{l}
\left({ }^{C} D_{a^{\rho+}}^{\alpha} v\right)(z)+\rho^{-\alpha} \varphi\left(z^{\frac{1}{\rho}}, T^{-1} v\right)=0, \quad a^{\rho}<z<b^{\rho}, \\
v\left(a^{\rho}\right)=v\left(b^{\rho}\right)=0 .
\end{array}\right.
$$

Using Lemma 2.2 with $A=a^{\rho}, B=b^{\rho}$, we obtain

$$
v(z)=\rho^{-\alpha} \int_{a^{\rho}}^{b^{\rho}} G(z, s) \varphi\left(s^{\frac{1}{\rho}}, T^{-1} v\right) d s, \quad a^{\rho} \leq z \leq b^{\rho} .
$$

Further, (2.5) and (3.1) yield

$$
|v(z)| \leq \rho^{-\alpha} \frac{\left[(\alpha-1)\left(b^{\rho}-a^{\rho}\right)\right]^{\alpha-1}}{\alpha^{\alpha} \Gamma(\alpha)}\left(\int_{a^{\rho}}^{b^{\rho}} w\left(s^{\frac{1}{\rho}}\right) d s\right)\left\|T^{-1} v\right\|_{\infty}, \quad a^{\rho} \leq z \leq b^{\rho},
$$

i.e.,

$$
\left|u\left(z^{\frac{1}{\rho}}\right)\right| \leq \rho^{-\alpha} \frac{\left[(\alpha-1)\left(b^{\rho}-a^{\rho}\right)\right]^{\alpha-1}}{\alpha^{\alpha} \Gamma(\alpha)}\left(\int_{a^{\rho}}^{b^{\rho}} w\left(s^{\frac{1}{\rho}}\right) d s\right)\|u\|_{\infty}, \quad a^{\rho} \leq z \leq b^{\rho},
$$


i.e.,

$$
|u(t)| \leq \rho^{-\alpha} \frac{\left[(\alpha-1)\left(b^{\rho}-a^{\rho}\right)\right]^{\alpha-1}}{\alpha^{\alpha} \Gamma(\alpha)}\left(\int_{a^{\rho}}^{b^{\rho}} w\left(s^{\frac{1}{\rho}}\right) d s\right)\|u\|_{\infty}, \quad a \leq t \leq b .
$$

Hence, we get

$$
\|u\|_{\infty} \leq \rho^{-\alpha} \frac{\left[(\alpha-1)\left(b^{\rho}-a^{\rho}\right)\right]^{\alpha-1}}{\alpha^{\alpha} \Gamma(\alpha)}\left(\int_{a^{\rho}}^{b^{\rho}} w\left(s^{\frac{1}{\rho}}\right) d s\right)\|u\|_{\infty} .
$$

Since $u$ is nontrivial, dividing by $\|u\|_{\infty}$, we obtain

$$
\int_{a^{\rho}}^{b^{\rho}} w\left(s^{\frac{1}{\rho}}\right) d s \geq \frac{\rho^{\alpha} \alpha^{\alpha} \Gamma(\alpha)}{\left[(\alpha-1)\left(b^{\rho}-a^{\rho}\right)\right]^{\alpha-1}} .
$$

Finally, using the change of variable $t=s^{\frac{1}{\rho}}, a^{\rho} \leq s \leq b^{\rho}$, (3.2) follows.

Further, we list some consequences following from Theorem 3.1.

Corollary 3.1 Let $u \in C^{2}([a, b]), a, b \in \mathbb{R}, 0<a<b$, be a nontrivial solution to

$$
\left\{\begin{array}{l}
\left({ }_{*}^{\rho} D_{a^{+}}^{\alpha} u\right)(t)+q(t)|u(t)|^{\lambda} \ln \left(1+|u(t)|^{1-\lambda}\right)=0, \quad a<t<b, \\
u(a)=u(b)=0,
\end{array}\right.
$$

where $\rho>0,1<\alpha<2,0<\lambda<1$ and $q \in C([a, b])$. Then

$$
\int_{a}^{b} t^{\rho-1}|q(t)| d t \geq \frac{\rho^{\alpha-1} \alpha^{\alpha} \Gamma(\alpha)}{\left[(\alpha-1)\left(b^{\rho}-a^{\rho}\right)\right]^{\alpha-1}} .
$$

Proof Observe that (3.3) is a special case of (1.1) with

$$
\varphi(t, h)=q(t)|h(t)|^{\lambda} \ln \left(1+|h(t)|^{1-\lambda}\right), \quad(t, h) \in[a, b] \times C([a, b]) .
$$

Moreover, using the inequality

$$
\ln (1+x) \leq x, \quad x \geq 0
$$

for all $(t, h) \in[a, b] \times C([a, b])$, we have

$$
\begin{aligned}
|\varphi(t, h)| & \leq|q(t)||h(t)|^{\lambda} \ln \left(1+|h(t)|^{1-\lambda}\right) \\
& \leq|q(t)||h(t)|^{\lambda}|h(t)|^{1-\lambda} \\
& =|q(t)||h(t)| \\
& \leq|q(t)|\|h\|_{\infty} .
\end{aligned}
$$

Hence, function $\varphi$ satisfies (3.1) with

$$
w(t)=|q(t)|, \quad a \leq t \leq b .
$$

Therefore, using Theorem 3.1 with $w$ given by (3.5), (3.4) follows. 
Corollary 3.2 (The case of a nonlocal source term) Let $u \in C^{2}([a, b]), a, b \in \mathbb{R}, 0<a<b$, be a nontrivial solution to

$$
\left\{\begin{array}{l}
\left({ }_{*}^{\rho} D_{a^{+}}^{\alpha} u\right)(t)+\frac{q(t)}{\Gamma(\theta)} \int_{a}^{t}(t-s)^{\theta-1} u(s) d s=0, \quad a<t<b, \\
u(a)=u(b)=0
\end{array}\right.
$$

where $\rho>0,1<\alpha<2, \theta>0$ and $q \in C([a, b])$. Then

$$
\int_{a}^{b} t^{\rho-1}(t-a)^{\theta}|q(t)| d t \geq \frac{\rho^{\alpha-1} \alpha^{\alpha} \Gamma(\theta+1) \Gamma(\alpha)}{\left[(\alpha-1)\left(b^{\rho}-a^{\rho}\right)\right]^{\alpha-1}} .
$$

Proof Observe that (3.6) is a special case of (1.1) with

$$
\varphi(t, h)=\frac{q(t)}{\Gamma(\theta)} \int_{a}^{t}(t-s)^{\theta-1} h(s) d s, \quad(t, h) \in[a, b] \times C([a, b]) .
$$

Moreover, for all $(t, h) \in[a, b] \times C([a, b])$, we have

$$
\begin{aligned}
|\varphi(t, h)| & \leq\|h\|_{\infty} \frac{|q(t)|}{\Gamma(\theta)} \int_{a}^{t}(t-s)^{\theta-1} d s \\
& =\frac{(t-a)^{\theta}|q(t)|}{\Gamma(\theta+1)}\|h\|_{\infty} .
\end{aligned}
$$

Hence, function $\varphi$ satisfies (3.1) with

$$
w(t)=\frac{(t-a)^{\theta}|q(t)|}{\Gamma(\theta+1)}, \quad a \leq t \leq b .
$$

Therefore, using Theorem 3.1 with $w$ given by (3.8), (3.7) follows.

Note that in the limit case $\theta \rightarrow 0^{+}$, (3.6) reduces to

$$
\left\{\begin{array}{l}
\left({ }_{*}^{\rho} D_{a^{+}}^{\alpha} u\right)(t)+q(t) u(t)=0, \quad a<t<b, \\
u(a)=u(b)=0 .
\end{array}\right.
$$

Therefore, passing to the limit as $\theta \rightarrow 0^{+}$in (3.7), we obtain the following result.

Corollary 3.3 Let $u \in C^{2}([a, b])$ be a nontrivial solution to (3.9). Then

$$
\int_{a}^{b} t^{\rho-1}|q(t)| d t \geq \frac{\rho^{\alpha-1} \alpha^{\alpha} \Gamma(\alpha)}{\left[(\alpha-1)\left(b^{\rho}-a^{\rho}\right)\right]^{\alpha-1}} .
$$

Remark 3.1 For $\rho=1$, (3.9) reduces to (1.6). Therefore, taking $\rho=1$ in (3.10), we obtain (1.7) (with a large inequality).

In the limit case $\rho \rightarrow 0^{+}$, by (2.1), (3.9) reduces to

$$
\left\{\begin{array}{l}
\left({ }^{\mathrm{CH}} D_{a^{+}}^{\alpha} u\right)(t)+q(t) u(t)=0, \quad a<t<b, \\
u(a)=u(b)=0
\end{array}\right.
$$


where ${ }^{\mathrm{CH}} D_{a^{+}}^{\alpha}$ is the Caputo-Hadamard fractional derivative of order $\alpha$ given by (2.2) with $n=2$. Therefore, passing to the limit as $\rho \rightarrow 0^{+}$in (3.10), we obtain the following result.

Corollary 3.4 Let $u \in C^{2}([a, b])$ be a nontrivial solution to (3.11). Then

$$
\int_{a}^{b} \frac{|q(t)|}{t} d t \geq \frac{\alpha^{\alpha} \Gamma(\alpha)}{[(\alpha-1)(\ln b-\ln a)]^{\alpha-1}}
$$

\section{A Lyapunov-type inequality for system (1.2)}

System (1.2) is investigated under the following assumptions:

(A1) The function

$$
\varphi:[a, b] \times C([a, b]) \times C([a, b]) \rightarrow \mathbb{R}
$$

is continuous and satisfies

$$
|\varphi(t, g, h)| \leq w_{11}(t)\|g\|_{\infty}+w_{12}(t)\|h\|_{\infty}, \quad(t, g, h) \in[a, b] \times C([a, b]) \times C([a, b]),
$$

where $w_{11}, w_{12} \in C([a, b])$ are positive functions.

(A2) The function

$$
\psi:[a, b] \times C([a, b]) \times C([a, b]) \rightarrow \mathbb{R}
$$

is continuous and satisfies

$$
|\psi(t, g, h)| \leq w_{21}(t)\|g\|_{\infty}+w_{22}(t)\|h\|_{\infty}, \quad(t, g, h) \in[a, b] \times C([a, b]) \times C([a, b]),
$$

where $w_{21}, w_{22} \in C([a, b])$ are positive functions.

We say that $(u, v) \in C^{2}([a, b]) \times C^{2}([a, b])$ is a nontrivial solution to (1.2) if $(u, v)$ satisfies $(1.2)$ and $(u, v) \not \equiv(0,0)$, where 0 is the zero function. Observe that by (A1) and (A2), $(0,0)$ is a solution to (1.2).

Our main result in this section is the following.

Theorem 4.1 Let $(u, v) \in C^{2}([a, b]) \times C^{2}([a, b])$ be a nontrivial solution to (1.2). Then

$$
\begin{aligned}
& \sqrt{\left(\int_{a}^{b}\left(w_{11}(t)-w_{22}(t)\right) t^{\rho-1} d t\right)^{2}+4\left(\int_{a}^{b} w_{21}(t) t^{\rho-1} d t\right)\left(\int_{a}^{b} w_{12}(t) t^{\rho-1} d t\right)} \\
& +\int_{a}^{b}\left(w_{11}(t)+w_{22}(t)\right) t^{\rho-1} d t \geq \frac{2 \rho^{\alpha-1} \alpha^{\alpha} \Gamma(\alpha)}{\left[(\alpha-1)\left(b^{\rho}-a^{\rho}\right)\right]^{\alpha-1}} .
\end{aligned}
$$

Proof Let $(u, v) \in C^{2}([a, b]) \times C^{2}([a, b])$ be a nontrivial solution to (1.2). We introduce the functions $(\bar{u}, \bar{v}) \in C^{2}\left(\left[a^{\rho}, b^{\rho}\right]\right) \times C^{2}\left(\left[a^{\rho}, b^{\rho}\right]\right)$ given by

$$
\bar{u}=T u \quad \text { and } \quad \bar{v}=T v .
$$


Using Lemma 2.1, we deduce that $(\bar{u}, \bar{v})$ is a solution to the system

$$
\begin{cases}\rho^{\alpha}\left({ }^{C} D_{a^{\rho+}}^{\alpha} \bar{u}\right)\left(t^{\rho}\right)+\varphi\left(t, T^{-1} \bar{u}, T^{-1} \bar{v}\right)=0, & a<t<b, \\ \rho^{\alpha}\left({ }^{C} D_{a^{\rho+}}^{\alpha} \bar{v}\right)\left(t^{\rho}\right)+\psi\left(t, T^{-1} \bar{u}, T^{-1} \bar{v}\right)=0, & a<t<b, \\ \bar{u}\left(a^{\rho}\right)=\bar{u}\left(b^{\rho}\right)=\bar{v}\left(a^{\rho}\right)=\bar{v}\left(b^{\rho}\right)=0 . & \end{cases}
$$

Using the change of variable $z=t^{\rho}, a \leq t \leq b$, we deduce that $(\bar{u}, \bar{v})$ is a solution to

$$
\begin{cases}\left({ }^{C} D_{a^{\rho+}}^{\alpha} \bar{u}\right)(z)+\rho^{-\alpha} \varphi\left(z^{\frac{1}{\rho}}, T^{-1} \bar{u}, T^{-1} \bar{v}\right)=0, & a^{\rho}<z<b^{\rho}, \\ \left({ }^{C} D_{a^{\rho+}}^{\alpha} \bar{v}\right)(z)+\rho^{-\alpha} \psi\left(z^{\frac{1}{\rho}}, T^{-1} \bar{u}, T^{-1} \bar{v}\right)=0, & a^{\rho}<z<b^{\rho}, \\ \bar{u}\left(a^{\rho}\right)=\bar{u}\left(b^{\rho}\right)=\bar{v}\left(a^{\rho}\right)=\bar{v}\left(b^{\rho}\right)=0 . & \end{cases}
$$

Using Lemma 2.2 with $A=a^{\rho}, B=b^{\rho}$, we obtain

$$
\bar{u}(z)=\rho^{-\alpha} \int_{a^{\rho}}^{b^{\rho}} G(z, s) \varphi\left(s^{\frac{1}{\rho}}, T^{-1} \bar{u}, T^{-1} \bar{v}\right) d s, \quad a^{\rho} \leq z \leq b^{\rho}
$$

and

$$
\bar{v}(z)=\rho^{-\alpha} \int_{a^{\rho}}^{b^{\rho}} G(z, s) \psi\left(s^{\frac{1}{\rho}}, T^{-1} \bar{u}, T^{-1} \bar{v}\right) d s, \quad a^{\rho} \leq z \leq b^{\rho} .
$$

Further, (2.5) and (A1) yield

$$
\begin{aligned}
|\bar{u}(z)| \leq & \rho^{-\alpha} \frac{\left[(\alpha-1)\left(b^{\rho}-a^{\rho}\right)\right]^{\alpha-1}}{\alpha^{\alpha} \Gamma(\alpha)} \\
& \times\left[\left(\int_{a^{\rho}}^{b^{\rho}} w_{11}\left(s^{\frac{1}{\rho}}\right) d s\right)\left\|T^{-1} \bar{u}\right\|_{\infty}+\left(\int_{a^{\rho}}^{b^{\rho}} w_{12}\left(s^{\frac{1}{\rho}}\right) d s\right)\left\|T^{-1} \bar{v}\right\|_{\infty}\right],
\end{aligned}
$$

for $a^{\rho} \leq z \leq b^{\rho}$, i.e.,

$$
\begin{aligned}
\left|u\left(z^{\frac{1}{\rho}}\right)\right| \leq & \rho^{-\alpha} \frac{\left[(\alpha-1)\left(b^{\rho}-a^{\rho}\right)\right]^{\alpha-1}}{\alpha^{\alpha} \Gamma(\alpha)} \\
& \times\left[\left(\int_{a^{\rho}}^{b^{\rho}} w_{11}\left(s^{\frac{1}{\rho}}\right) d s\right)\left\|T^{-1} \bar{u}\right\|_{\infty}+\left(\int_{a^{\rho}}^{b^{\rho}} w_{12}\left(s^{\frac{1}{\rho}}\right) d s\right)\left\|T^{-1} \bar{v}\right\|_{\infty}\right],
\end{aligned}
$$

for $a^{\rho} \leq z \leq b^{\rho}$, which implies that

$$
\|u\|_{\infty} \leq A_{11}\|u\|_{\infty}+A_{12}\|v\|_{\infty}
$$

where

$$
A_{1 j}=\rho^{1-\alpha} \frac{\left[(\alpha-1)\left(b^{\rho}-a^{\rho}\right)\right]^{\alpha-1}}{\alpha^{\alpha} \Gamma(\alpha)} \int_{a}^{b} w_{1 j}(t) t^{\rho-1} d t, \quad j=1,2 .
$$

Using (A2) and a similar argument as above, we get

$$
\|v\|_{\infty} \leq A_{21}\|u\|_{\infty}+A_{22}\|v\|_{\infty}
$$


where

$$
A_{2 j}=\rho^{1-\alpha} \frac{\left[(\alpha-1)\left(b^{\rho}-a^{\rho}\right)\right]^{\alpha-1}}{\alpha^{\alpha} \Gamma(\alpha)} \int_{a}^{b} w_{2 j}(t) t^{\rho-1} d t, \quad j=1,2 .
$$

Further, combining (4.2) with (4.3), we obtain

$$
0_{\mathbb{R}^{2}} \preceq_{2} \vec{x} \preceq_{2} A \vec{x},
$$

where $A=\left(A_{i j}\right)_{1 \leq i, j \leq 2} \in \mathcal{M}_{2}\left(\mathbb{R}_{+}\right)$and $\vec{x}=\left(\begin{array}{l}\|u\|_{\infty} \\ \|v\|_{\infty}\end{array}\right)$. Since $(u, v)$ is a nontrivial solution to (1.2), we have $\vec{x} \neq 0_{\mathbb{R}^{2}}$. Therefore, by Lemma 2.5 , we have

$$
r(A) \geq 1 .
$$

Let $P_{A}$ be the characteristic polynomial of the matrix $A$, i.e.,

$$
P_{A}(\lambda)=\lambda^{2}-\operatorname{tr}(A) \lambda+\operatorname{det}(A), \quad \lambda \in \mathbb{C},
$$

where $\operatorname{tr}(A)$ is the trace of $A$ and $\operatorname{det}(A)$ is its determinant. Then the discriminant of $P_{A}$ is given by

$$
\Delta\left(P_{A}\right)=\left(A_{11}-A_{22}\right)^{2}+4 A_{21} A_{12} .
$$

Note that since $A \in \mathcal{M}_{2}\left(\mathbb{R}_{+}\right)$, we have $\Delta\left(P_{A}\right) \geq 0$. Therefore, the eigenvalues of the matrix $A$ are given by

$$
\lambda_{1}(A)=\frac{A_{11}+A_{22}+\sqrt{\left(A_{11}-A_{22}\right)^{2}+4 A_{21} A_{12}}}{2}
$$

and

$$
\lambda_{2}(A)=\frac{A_{11}+A_{22}-\sqrt{\left(A_{11}-A_{22}\right)^{2}+4 A_{21} A_{12}}}{2} .
$$

Observe that $\lambda_{1}(A) \geq \lambda_{2}(A)$. We discuss two cases.

Case 1. $A_{11} A_{22} \geq A_{21} A_{12}$.

In this case, we have $\lambda_{2}(A) \geq 0$, which yields

$$
r(A)=\lambda_{1}(A) .
$$

Case 2. $A_{11} A_{22}<A_{21} A_{12}$.

In this case, we have

$$
\lambda_{1}(A) \geq\left|\lambda_{2}(A)\right|=\frac{\sqrt{\left(A_{11}-A_{22}\right)^{2}+4 A_{21} A_{12}}-A_{11}-A_{22}}{2},
$$

which implies that

$$
r(A)=\lambda_{1}(A) .
$$


Therefore, we proved that in both cases, we have

$$
r(A)=\frac{A_{11}+A_{22}+\sqrt{\left(A_{11}-A_{22}\right)^{2}+4 A_{21} A_{12}}}{2} .
$$

Finally, combining (4.4) with (4.5), (4.1) follows.

Further, we list some special cases following from Theorem 4.1.

Let us consider the system

$$
\left\{\begin{array}{l}
\left({ }_{*}^{\rho} D_{a^{+}}^{\alpha} u\right)(t)+\mu(t) u(t)+v(t) v(t)=0, \quad a<t<b \\
\left({ }_{*}^{\rho} D_{a^{+}}^{\alpha} v\right)(t)+\chi(t) u(t)+\mu(t) v(t), \quad a<t<b \\
u(a)=u(b)=v(a)=v(b)=0
\end{array}\right.
$$

where $a, b \in \mathbb{R}, 0<a<b, \rho>0,1<\alpha<2$ and $\mu, v, \chi \in C([a, b])$. Observe that (4.6) is a special case of (1.2) with

$$
\varphi(t, g, h)=\mu(t) g(t)+v(t) h(t), \quad(t, g, h) \in[a, b] \times C([a, b]) \times C([a, b])
$$

and

$$
\psi(t, g, h)=\chi(t) g(t)+\mu(t) h(t), \quad(t, g, h) \in[a, b] \times C([a, b]) \times C([a, b]) .
$$

Note that the function $\varphi$ satisfies (A1) with

$$
w_{11}=|\mu| \quad \text { and } \quad w_{12}=|\nu| .
$$

Moreover, the function $\psi$ satisfies (A2) with

$$
w_{21}=|\chi| \quad \text { and } \quad w_{22}=w_{11} .
$$

Hence, using Theorem 4.1, we obtain the following result.

Corollary 4.1 Let $(u, v) \in C^{2}([a, b]) \times C^{2}([a, b])$ be a nontrivial solution to (4.6). Then

$$
\sqrt{\left(\int_{a}^{b}|\chi(t)| t^{\rho-1} d t\right)\left(\int_{a}^{b}|v(t)|(t) t^{\rho-1} d t\right)}+\int_{a}^{b}|\mu(t)| t^{\rho-1} d t \geq \frac{\rho^{\alpha-1} \alpha^{\alpha} \Gamma(\alpha)}{\left[(\alpha-1)\left(b^{\rho}-a^{\rho}\right)\right]^{\alpha-1}} .
$$

Let us consider the system

$$
\left\{\begin{array}{l}
\left({ }_{*}^{\rho} D_{a^{+}}^{\alpha} u\right)(t)+\mu(t) u(t)+v(t) v(t)=0, \quad a<t<b, \\
\left({ }_{*}^{\rho} D_{a^{+}}^{\alpha} v\right)(t)+\chi(t) v(t), \quad a<t<b, \\
u(a)=u(b)=v(a)=v(b)=0
\end{array}\right.
$$

where $a, b \in \mathbb{R}, 0<a<b, \rho>0,1<\alpha<2$ and $\mu, v, \chi \in C([a, b])$. Observe that (4.7) is a special case of (1.2) with

$$
\varphi(t, g, h)=\mu(t) g(t)+v(t) h(t), \quad(t, g, h) \in[a, b] \times C([a, b]) \times C([a, b])
$$


and

$$
\psi(t, g, h)=\chi(t) h(t), \quad(t, g, h) \in[a, b] \times C([a, b]) \times C([a, b]) .
$$

Note that the function $\varphi$ satisfies (A1) with

$$
w_{11}=|\mu| \quad \text { and } \quad w_{12}=|\nu| .
$$

Moreover, the function $\psi$ satisfies (A2) with

$$
w_{21} \equiv 0 \quad \text { and } \quad w_{22}=|\chi|
$$

Hence, using Theorem 4.1, we obtain the following result.

Corollary 4.2 Let $(u, v) \in C^{2}([a, b]) \times C^{2}([a, b])$ be a nontrivial solution to (4.7). Then

$$
\left|\int_{a}^{b}(|\mu(t)|-|\chi(t)|) t^{\rho-1} d t\right|+\int_{a}^{b}(|\mu(t)|+|\chi(t)|) t^{\rho-1} d t \geq \frac{2 \rho^{\alpha-1} \alpha^{\alpha} \Gamma(\alpha)}{\left[(\alpha-1)\left(b^{\rho}-a^{\rho}\right)\right]^{\alpha-1}} .
$$

\section{Conclusions}

In this contribution, nonlinear fractional differential equations involving Caputo-type fractional derivatives have been considered. Necessary conditions for the existence of nontrivial solutions to the considered problems have been obtained. We have discussed both cases: the case of an equation and the case of a coupled system. For each case, a Lyapunov-type inequality has been established. We expect that the proposed approaches and techniques used in this paper can be adapted to study other fractional boundary value problems.

\section{Acknowledgements}

The second author extends his appreciation to Distinguished Scientist Fellowship Program (DSFP) at King Saud University (Saudi Arabia). The third author acknowledges the support by National Natural Science Foundation of China (11671339).

Funding

This work was partially supported by DSFP at King Saud University.

Availability of data and materials

Not applicable.

Competing interests

The authors declare that they have no competing interests.

Authors' contributions

All authors contributed equally in this work. All authors read and approved the final manuscript.

\section{Author details}

'Department of Mathematics, College of Science, King Saud University, Riyadh, Saudi Arabia. ${ }^{2}$ Faculty of Information Technology, Macau University of Science and Technology, Macau, China. ${ }^{3}$ Faculty of Mathematics and Computational Science, Xiangtan University, Xiangtan, China.

\section{Publisher's Note}

Springer Nature remains neutral with regard to jurisdictional claims in published maps and institutional affiliations. 


\section{References}

1. Agarwal, R.P., Jleli, M., Samet, B.: On de la Vallée Poussin-type inequalities in higher dimension and applications. Appl. Math. Lett. 86, 264-269 (2018)

2. Borg, G.: On a Liapunoff criterion of stability. Am. J. Math. 71, 67-70 (1949)

3. Cabrera, I., Lopez, B., Sadarangani, K.: Lyapunov type inequalities for a fractional two-point boundary value problem. Math. Methods Appl. Sci. 40, 3409-3414 (2017)

4. Cañada, A., Villegas, S.: Lyapunov inequalities for Neumann boundary conditions at higher eigenvalues. J. Eur. Math. Soc. 12, 163-178 (2010)

5. Dahiya, R.S., Singh, B.: A Liapunov inequality and nonoscillation theorem for a second order nonlinear differential-difference equations. J. Math. Phys. Sci. 7, 163-170 (1973)

6. Das, A.M., Vatsala, A.S.: Green function for $n-n$ boundary value problem and an analogue of Hartman's result. J. Math. Anal. Appl. 51, 670-677 (1975)

7. Dhar, S., Kong, Q., McCabe, M.: Fractional boundary value problems and Lyapunov-type inequalities with fractional integral boundary conditions. Electron. J. Qual. Theory Differ. Equ. 2016, 43 (2016)

8. Dhar, S., Kong, Q.: Lyapunov-type inequalities for $\alpha$-th order fractional differential equations with $2<\alpha \leq 3$ and fractional boundary conditions. Electron. J. Differ. Equ. 2017, 203, 1-15 (2017)

9. Dym, H.: Linear Algebra in Action. Graduate Studies in Mathematics, vol. 78. Am. Math. Soc., Providence (2007)

10. Eliason, S.B.: A Liapunov inequality for a certain second order nonlinear differential equation. J. Lond. Math. Soc. 2 461-466 (1970)

11. Ferreira, R.A.C.: A Lyapunov-type inequality for a fractional boundary value problem. Fract. Calc. Appl. Anal. 16(4), 978-984 (2013)

12. Ferreira, R.A.C.: On a Lyapunov-type inequality and the zeros of a certain Mittag-Leffler function. J. Math. Anal. Appl. 412, 1058-1063 (2014)

13. Jleli, M., Kirane, M., Samet, B.: On Lyapunov-type inequalities for a certain class of partial differential equations. Appl. Anal. (2018). https://doi.org/10.1080/00036811.2018.1484909

14. Jeli, M., Kirane, M., Samet, B.: Hartman-Wintner-type inequality for a fractional boundary value problem via a fractional derivative with respect to another function. Discrete Dyn. Nat. Soc. 2017, 5123240 (2017) (8 pages)

15. Jleli, M., Nieto, J., Samet, B.: Lyapunov-type inequalities for a higher order fractional differential equation with fractional integral boundary conditions. Electron. J. Qual. Theory Differ. Equ. 2017, 16 (2017)

16. Jleli, M., Ragoub, L., Samet, B.: A Lyapunov-type inequality for a fractional differential equation under a Robin boundary condition. J. Funct. Spaces 2015, 468536 (2015) (5 pages)

17. Jleli, M., Samet, B.: Lyapunov type inequalities for a fractional differential equation with mixed boundary value problems. Math. Inequal. Appl. 18, 443-451 (2015)

18. Jleli, M., Samet, B.: Lyapunov-type inequalities for fractional boundary-value problems. Electron. J. Differ. Equ. 2015,88 1-11 (2015)

19. Jleli, M., Samet, B.: Existence of positive solutions to a coupled system of fractional differential equations. Math. Methods Appl. Sci. 38, 1014-1031 (2015)

20. Katugampola, U.N.: New approach to a generalized fractional integral. Appl. Math. Comput. 218(3), 860-865 (2011)

21. Katugampola, U.N.: A new approach to generalized fractional derivatives. Bull. Math. Anal. Appl. 6(4), 1-15 (2014)

22. Kwong, M.K.: On Lyapunov's inequality for disfocality. J. Math. Anal. Appl. 83, 486-494 (1981)

23. Lyapunov, A.M.: Problème geńeŕal de la stabilité du mouvement. Ann. Fac. Sci. Univ. Toulouse Sci. Math. Sci. Phys. 2 203-407 (1907)

24. Napoli, P.L., Pinasco, J.P.: Estimates for eigenvalues of quasilinear elliptic systems. J. Differ. Equ. 227, 102-115 (2006)

25. Oliveira, D.S., de Oliveira, E.C.: On a Caputo-type fractional derivative. Adv. Pure Appl. Math. (2018). https://doi.org/10.1515/apam-2017-0068

26. O'Regan, D., Samet, B.: Lyapunov-type inequalities for a class of fractional differential equations. J. Inequal. Appl. 2015, 247 (10 pages) (2015)

27. Rong, J., Bai, C.Z.: Lyapunov-type inequality for a fractional differential equations with fractional boundary value problems. Adv. Differ. Equ. 2015, 82 (10 pages) (2015)

28. Pachpatte, B.G.: On Liapunov-type inequalities for certain higher order differential equations. J. Math. Anal. Appl. 195, 527-536 (1995)

29. Parhi, N., Panigrahi, S.: Liapunov-type inequality for higher order differential equations. Math. Slovaca 52(1), 31-46 (2002)

30. Reid, W.T.: Interrelations between a trace formula and Liapunov inequalities. J. Differ. Equ. 23, 448-458 (1977)

31. Samko, S.G., Kilbas, A.A., Marichev, O.I.: Fractional Integrals and Derivatives. Gordon \& Breach, Yverdon (1993)

32. Wintner, A.: On the nonexistence of conjugate points. Am. J. Math. 73, 368-380 (1951)

33. Yang, $X .:$ On Liapunov-type inequality for certain higher-order differential equations. Appl. Math. Comput. 134, 307-317 (2003)

34. Zhang, M., Li, W.: A Lyapunov-type stability criterion using $L^{\alpha}$ norms. Proc. Am. Math. Soc. 130, 3325-3333 (2002) 